\title{
Au-Ag Core-Shell Nanospheres for Surface-Enhanced Raman Scattering Detection of Sudan I and Sudan II in Chili Powder
}

\author{
Lu Pei, Yiming Ou, Wansong Yu, Yuxia Fan, Yiqun Huang, and Keqiang Lai \\ College of Food Science and Technology, Shanghai Ocean University, No. 999 Hucheng Huan Road, LinGang New City, \\ Shanghai, 201306, China
}

Correspondence should be addressed to Keqiang Lai; kqlai@shou.edu.cn

Received 27 March 2015; Accepted 9 July 2015

Academic Editor: Renyun Zhang

Copyright (C) $2015 \mathrm{Lu}$ Pei et al. This is an open access article distributed under the Creative Commons Attribution License, which permits unrestricted use, distribution, and reproduction in any medium, provided the original work is properly cited.

Au-Ag core-shell (Au@Ag) bimetallic nanospheres synthesized by a facile seed-growth method are proposed as a substrate for surface-enhanced Raman spectroscopy (SERS) to detect azo-group dyes including Sudan I and Sudan II. Au@Ag nanospheres with a series of particle sizes (diameter: $30-120 \mathrm{~nm}$ ) and silver shell thicknesses $(6-51 \mathrm{~nm})$ were synthesized and compared for their morphological and optical properties to obtain optimum enhancement effect. Normal Raman, SERS, infrared, and ultravioletvisible were used to investigate the optical absorption properties of Sudan I and Sudan II as well as the enhancement mechanism of Au@Ag substrates. The nanospheres with particle size of $73 \pm 6 \mathrm{~nm}$ in diameter and silver layer of $27 \pm 2 \mathrm{~nm}$ resulted in the highest enhancement effect and could be used to detect Sudan I and Sudan II standard solutions at levels as low as 0.4 and $0.1 \mathrm{mg} / \mathrm{L}$, respectively. Moreover, Sudan I and Sudan II in chili powder could be detected at 0.6 and $0.4 \mathrm{mg} / \mathrm{kg}$, respectively. Sudan I and Sudan II with similar structures in complicated food matrices could be distinguished through applying principal component analysis, indicating good selectivity of the SERS method for detection of banned additives in food stuffs at trace levels.

\section{Introduction}

Sudan dyes are a group of synthetic aromatic compounds containing azo-group $(-\mathrm{N}=\mathrm{N}-)$ as shown in Figure 1, which are commonly used in hydrocarbon solvents, oils, waxes, textiles, shoes, and plastics to provide an orange-red appearance. Some of the Sudan dyes, such as Sudan I, was classified as class three carcinogen and can be metabolized by intestinal bacteria to produce carcinogenic compounds $[1,2]$. The use of Sudan dyes as coloring agents in foods has been banned in the USA, Australia, Canada, China, and the European Union (EU) since 1995, but the abuse of Sudan dyes in food is not uncommon because of the low cost and wide availability of these dyes [3]. High performance liquid chromatography with the ultraviolet-visible (UV-vis), or diode array detection, or electrochemical detection has been used as the main method for detecting the presence of Sudan dyes in foodstuffs, although rapid methods such as enzyme-linked immunosorbent assay have also been reported [4]. Timeconsumption, lack of sensitivity or accuracy, and/or high cost have always been the issues tied to the current analytical methods.
Surface-enhanced Raman scattering or surface-enhanced Raman spectroscopy (SERS) emerges as one of the most potent analytical tools in various fields because of its rapidity, high sensitivity, and selectivity for molecular detection $[5,6]$. The most important factor for successful applications of SERS is the use of SERS substrate with proper nanostructured metal surface that allows for effective adsorption of the targeted molecules onto the substrate surface and results in high Raman scattering enhancement and reproducibility [7]. The use of Au@Ag core-shell bimetallic nanoparticles (NPs) as SERS substrate has both the advantages of high enhancement effects of Ag NPs as well as homogeneity and stability of $\mathrm{Au}$ NPs $[8,9]$. In addition, the core-shell bimetallic structure offers the possibility of a tunable localized surface plasmon resonance (LSPR) that gives rise to SERS by varying the size of the Au core or/and the thickness of the Ag shell, which makes Au@Ag NPs an ideal candidate as substrate for SERS studies $[10,11]$.

The objective of this study was to apply easy-to-prepare Au@Ag substrates with superior SERS performances (high sensitivity, reproducibility, and selectivity) to quickly detect 


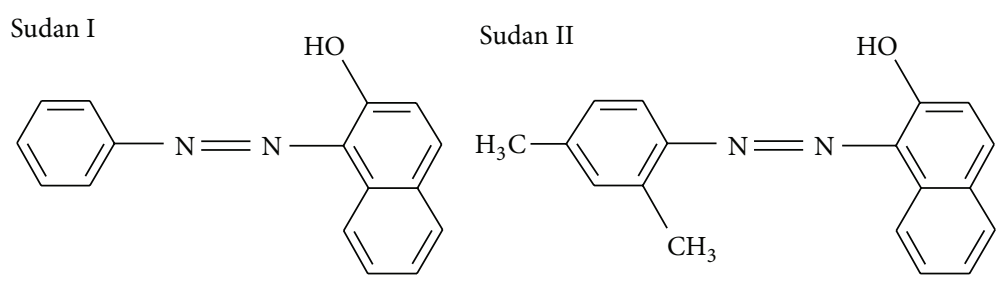

FIGURE 1: The molecular structures of Sudan I and Sudan II.

trace levels of Sudan I and Sudan II. To obtain appropriate particle size and thickness of silver shell for high sensitive SERS effect, Au@Ag substrates with a series of particle sizes and Ag shell thicknesses were synthesized and compared for their morphological and optical properties. Four spectroscopic techniques including normal Raman, SERS, infrared (IR), and UV-vis were used to investigate the optical absorption properties of Sudan I and Sudan II as well as the enhancement mechanism of Au@Ag substrates. Principal component analysis (PCA) was used as an exploratory tool to elucidate the spectral differentiation between Sudan I and II.

\section{Materials and Methods}

2.1. Synthesis of Au@Ag Nanoparticleswith Different Sizes. Colloidal Au nanospheres with diameter of $18 \pm 2 \mathrm{~nm}$ were synthesized as seeds according to the Frens method [12]. Asprepared Au colloid and L-ascorbic acid $(1.2 \mathrm{~mL}, 0.1 \mathrm{~mol} / \mathrm{L})$ were added in a glass vial, stirring continuously. $\mathrm{AgNO}_{3}$ solution was added drop-by-drop into this vial and the final mixture was continuously stirred for 5 min to form Au@Ag NPs. The sizes of Au@Ag NPs were controlled by using different amount $(0.45,0.9,1.5$ and $4.5 \mathrm{~mL})$ of $\mathrm{Au}$ seeds and varying the concentration $(0.5,1$ and $2 \mathrm{mmol} / \mathrm{L})$ of silver nitrate during silver coating process. The color of Au@Ag NPs ranged from orange to cream when the amount of Au seeds used for silver coating decreased or the concentration of silver nitrate increased.

The surface morphology of Au@Ag NPs were analyzed with transmission electron microscopy (TEM, JEM-2100F, JEOL Ltd., Tokyo, Japan) and the average particle sizes was calculated based upon the diameters of 100 particles. In addition, the optical absorption of Au NPs and Au@Ag NPs were analyzed with a UV-vis absorbance spectroscopy (UV3000PC, MAPADA Instruments Ltd., Shanghai, China) over a range of $300-800 \mathrm{~nm}$.

2.2. Preparation of Standard Solutions. Sudan I and Sudan II purchased from Sigma-Aldrich ( $\geq 96 \%$; Sigma-Aldrich Chemicals, St. Louis, MO) were dissolved into acetonitrile (HPLC reagent; Sigma-Aldrich Chemicals, St. Louis, MO) aqueous solution $(\mathrm{v} / \mathrm{v}=1: 1)$ to prepare a series of standard solutions $(0.1-1.0 \mathrm{mg} / \mathrm{L})$ for each dye.

2.3. Chili Samples Pretreatment. Chili powder was purchased from a local supermarket in Shanghai, China. Sudan I and Sudan II were extracted from chili powder based on an international method released by the Government Chemist of liquid chromatography, and the recovery rate was about $80-110 \%$. Briefly, $1 \mathrm{~g}$ of chili powder was vigorously mixed with $10 \mathrm{~mL}$ of acetonitrile for $2 \mathrm{~min}$. The mixture was then shaken $(280$ times/min) in a vibrator for $30 \mathrm{~min}$ at room temperature and centrifuged at $5000 \mathrm{rpm}$ for $10 \mathrm{~min}$. The supernatants were combined after a second centrifugation with $10 \mathrm{~mL}$ of acetonitrile added and then evaporated to dryness with a rotary evaporator (R206B, Shanghai SENCO Technology Ltd., Shanghai, China) at $40^{\circ} \mathrm{C}$. Following this, the residue was reconstituted in $1 \mathrm{~mL}$ of acetonitrile and further purified using $\mathrm{C} 18$ cartridge for solid phase extraction (500 mg, $3 \mathrm{~mL}$; Supelco, Bellefonte, PA, USA). The extracts were finally filtered through $0.45 \mu \mathrm{m}$ pore size of syringe filters and spiked with Sudan I [0-1 mg/kg] or Sudan II [0$1 \mathrm{mg} / \mathrm{kg}$. The extracts spiked with Sudan dyes were kept at refrigerator temperature before further use.

2.4. Spectral Measurements. Infrared (IR) spectra of Sudan dyes were obtained using a Nicolet 6700 FTIR spectrometer (Thermo Fisher Scientific Inc., Waltham, MA, USA) equipped with a DTGS detector operating at room temperature. Sudan I or Sudan II was ground with $\mathrm{KBr}$ powder and pressed into a thin pellet. The spectrum of the pellet was obtained in transmittance mode with spectral resolution of $4 \mathrm{~cm}^{-1}$ and was an average of 32 scans.

A Nicolet DXR microscopy Raman spectrometer (Thermo Fisher Scientific Inc., Waltham, MA, USA) with a $633 \mathrm{~nm} \mathrm{He}-\mathrm{Ne}$ laser was employed to acquire Raman and SERS spectra. All spectra were collected at $5 \mathrm{~mW}$, $20 \mathrm{x}$ objective (Olympus) with a slit width of $50 \mathrm{~cm}^{-1}$. The accumulation time was $2 \mathrm{~s}$ for each of the five scans acquired for a sample.

To assemble SERS substrates, Au@Ag NPs were concentrated 3 times and then mixed with standard solution $(\mathrm{v} / \mathrm{v}=$ $1: 1)$ or chili sample extract $(\mathrm{v} / \mathrm{v}=2: 1)$. The mixture $(5 \mu \mathrm{L})$ was pipetted onto a microscope glass slide, and SERS spectra were acquired after evaporation of the solvent. To ensure the reproducibility of a SERS spectrum, the average of a total of 30 spectra collected from 10 different spots at each of the three batches of substrates was used as the final spectrum for a sample.

2.5. Principal Component Analysis (PCA). Multivariate PCA analysis [DeLight 3.2 (DSquared Development Inc., LaGrande, OR, USA)] was used to differentiate spectral data for Sudan I and Sudan II extracts. The results of the data analysis reflect the selectivity of SERS method for different analytes. In order to ensure that the result of PCA was 


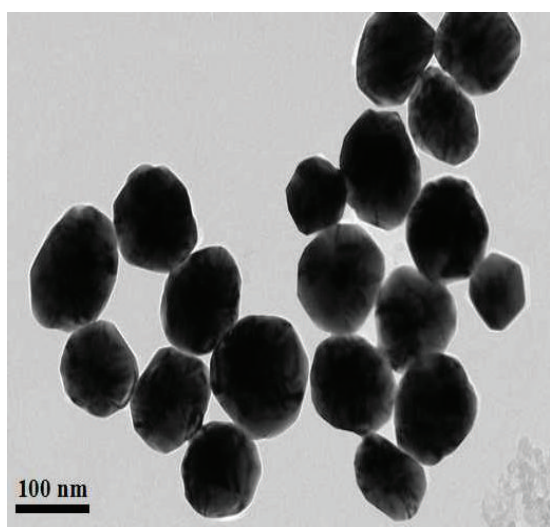

(a)

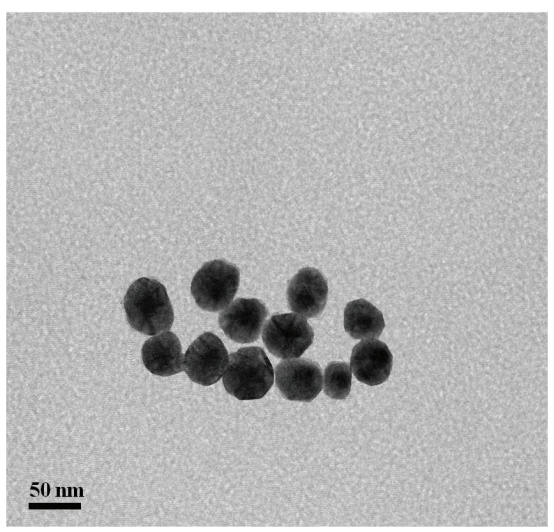

(d)

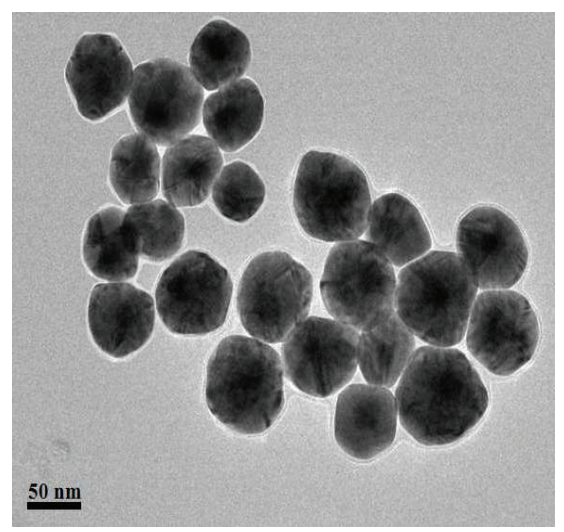

(b)

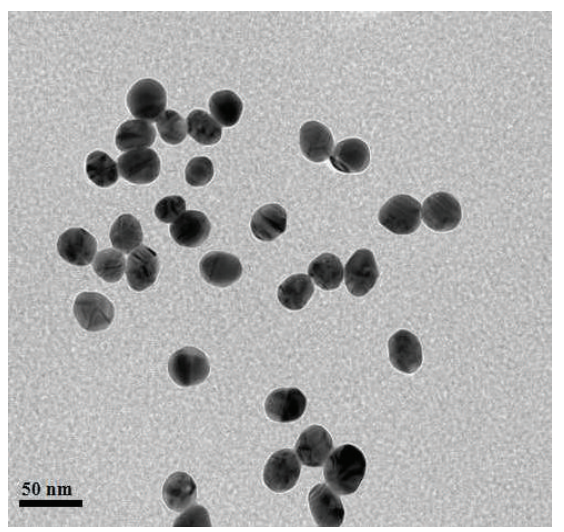

(e)

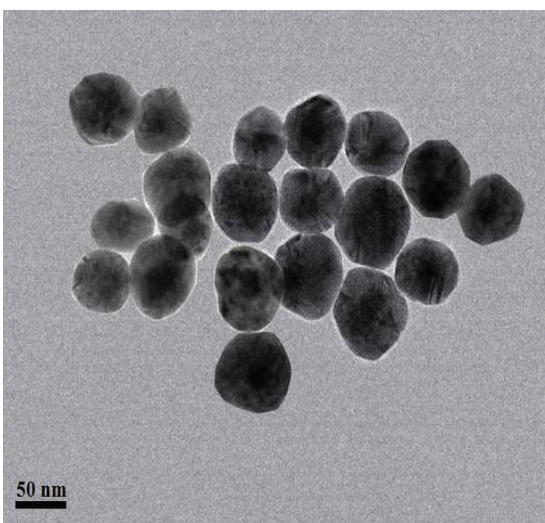

(c)

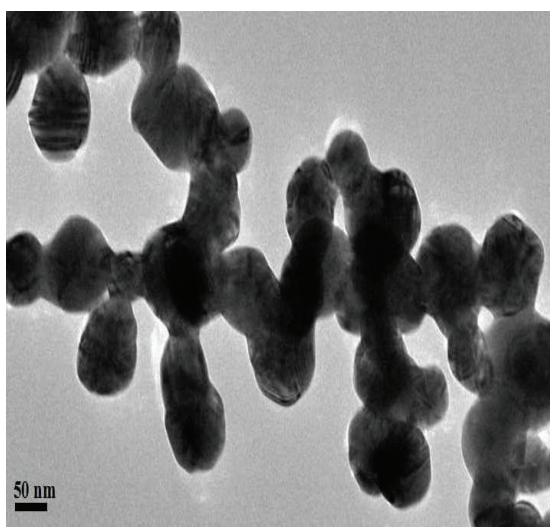

(f)

FIGURE 2: TEM images of Au@Ag nanoparticles synthesized with $1 \mathrm{mmol} / \mathrm{L} \mathrm{AgNO}_{3}$ solution and different amounts of Au seeds including (a) $0.45 \mathrm{~mL}$, (b) $0.9 \mathrm{~mL}$, (c) $1.5 \mathrm{~mL}$, and (d) $4.5 \mathrm{~mL}$ and synthesized with $0.9 \mathrm{~mL}$ Au seeds and different concentrations of $\mathrm{AgNO}_{3}$ solutions including (e) $0.5 \mathrm{mmol} / \mathrm{L}$ and (f) $2 \mathrm{mmol} / \mathrm{L}$.

reliable and the spectra were representative, three different concentrations of extracts were used for Sudan I ( $0.4,0.5$ and $1 \mathrm{mg} / \mathrm{kg}$ ) and for Sudan II $(0.6,0.8$ and $1 \mathrm{mg} / \mathrm{kg})$, respectively.

\section{Results and Discussion}

3.1. Morphological and Optical Characteristics of Au@Ag NPs. $\mathrm{Au} @ \mathrm{Ag}$ nanoparticles were prepared by deposition of Ag through chemical reduction in the presence of $\mathrm{Au}$ seeds. By adjusting the amounts of $\mathrm{AgNO}_{3}$ solution and $\mathrm{Au}$ seeds used, the thickness of the shell and thus the size of bimetallic particles could be controlled easily. As the amount of $\mathrm{Au}$ colloid used was increased from $0.45 \mathrm{~mL}$ to $0.9 \mathrm{~mL}, 1.5 \mathrm{~mL}$, and $4.5 \mathrm{~mL}$, with the use of $1 \mathrm{mmol} / \mathrm{L} \mathrm{AgNO}_{3}$ for silver coating, the average diameters of the resulted bimetallic NPs were decreased from $120 \pm 11 \mathrm{~nm}$ to $73 \pm 6 \mathrm{~nm}, 61 \pm 5 \mathrm{~nm}$, and $50 \pm 4 \mathrm{~nm}$, respectively, while the corresponding Ag shell thickness was about $51 \mathrm{~nm}, 27 \mathrm{~nm}, 21 \mathrm{~nm}$, and $16 \mathrm{~nm}$, respectively (Figures 2(a)-2(d)). This is because, with increased numbers of Au seeds, the amounts of Ag precipitated onto each $\mathrm{Au}$ nanoparticle would decrease, resulting in smaller Au@Ag nanoparticles [13, 14]. Similarly, the thickness of silver shells could also be controlled by varying the $\mathrm{AgNO}_{3}$ concentration while keeping the amount of Au seeds constant during silver coating process, and a higher $\mathrm{AgNO}_{3}$ concentration led to larger bimetallic NPs (Figures 2(b), 2(e), and 2(f)). However, when the concentration of $\mathrm{AgNO}_{3}$ solution was too high, such as $2 \mathrm{mmol} / \mathrm{L}$, some necklace-like chains were formed due to excessive $\mathrm{Ag}$ reduced from $\mathrm{Ag}^{+}$. The excessive Ag was deposited in the gaps between Au@Ag NPs and thus produced adhesion between particles rather than continuously coated on the surface of Ag shells to form larger particle sizes [15].

The Au core and Ag shell structure of Au@Ag nanoparticles was confirmed with the use of scanning TEM-high angle annular dark field (STEM-HAADF) imaging and energy dispersive spectroscopy (EDS) elemental mapping (Figure 3). The relative position of $\mathrm{Au}$ and $\mathrm{Ag}$ in a NP can be directly observed as a brighter, denser outer shell and a darker, sparser inner region in the STEM-HAADF image due to their different atomic number. The EDS mapping results indicated that the $\mathrm{Au}$ is in the center of the particle area as the core (in red), while the Ag is located on the outside as the shell (in green).

The surface plasmon resonance (SPR) peaks for colloidal Au seeds and Au@Ag bimetallic NPs synthesized using different amounts of Au seeds are shown in Figure 4. 

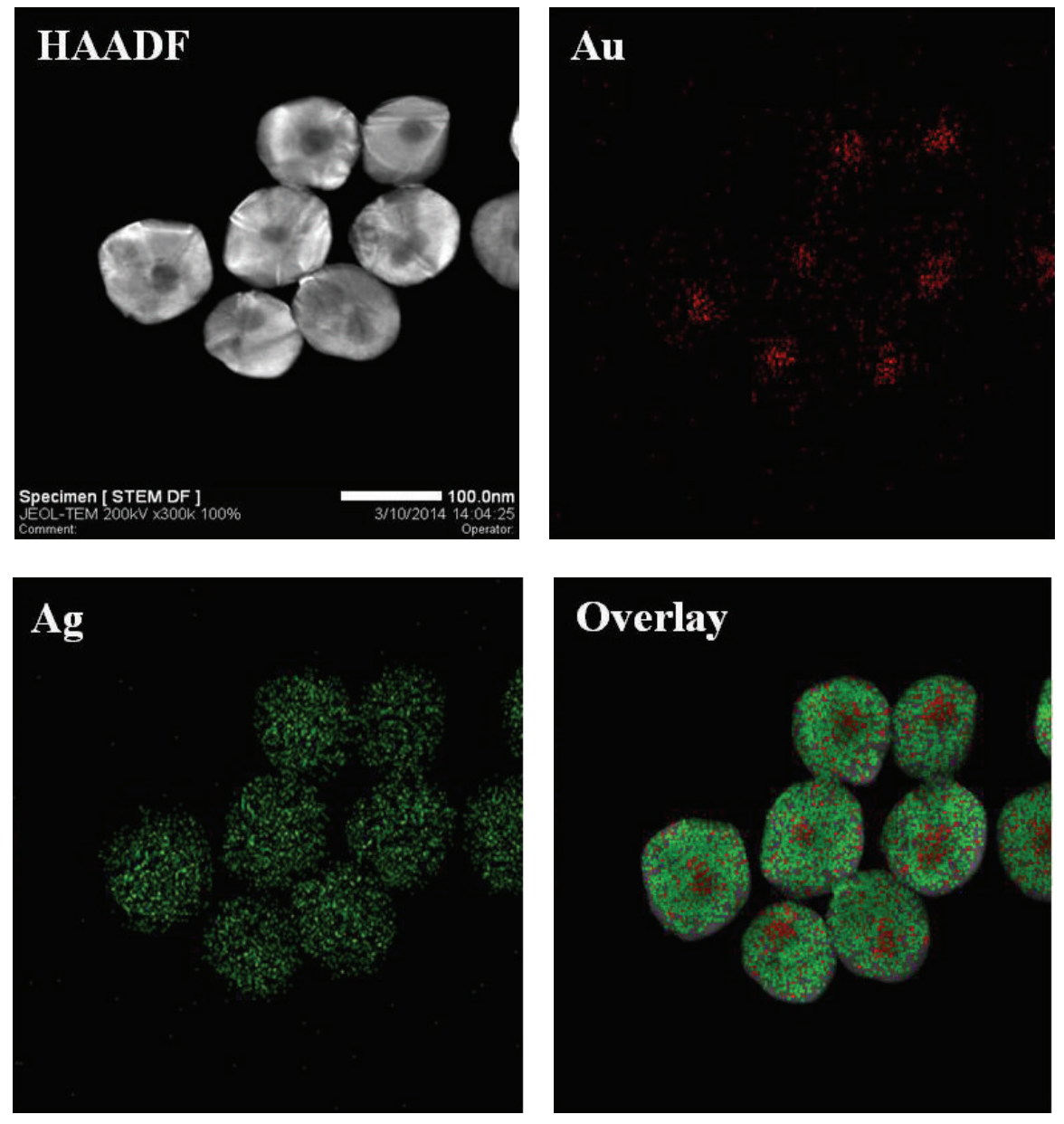

Figure 3: STEM-HAADF and EDS elemental mapping images for Au L map and Ag L map and an overlay of the Au and Ag maps of Au@Ag NPs (particle sizes about $120 \mathrm{~nm}$ ). The scale bar in STEM-HAADF applies to all images.

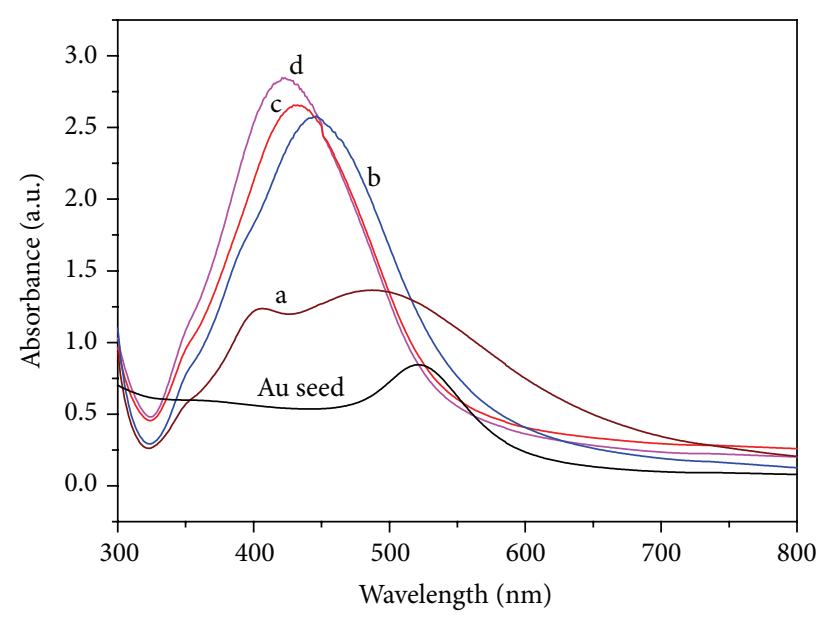

FIGURE 4: UV-vis spectra of colloidal Au seeds and Au@Ag NPs synthesized with $1 \mathrm{mmol} / \mathrm{L} \mathrm{AgNO}_{3}$ solution and various amounts of Au seeds including (a) $0.45 \mathrm{~mL}$, (b) $0.9 \mathrm{~mL}$, (c) $1.5 \mathrm{~mL}$, and (d) $4.5 \mathrm{~mL}$. 

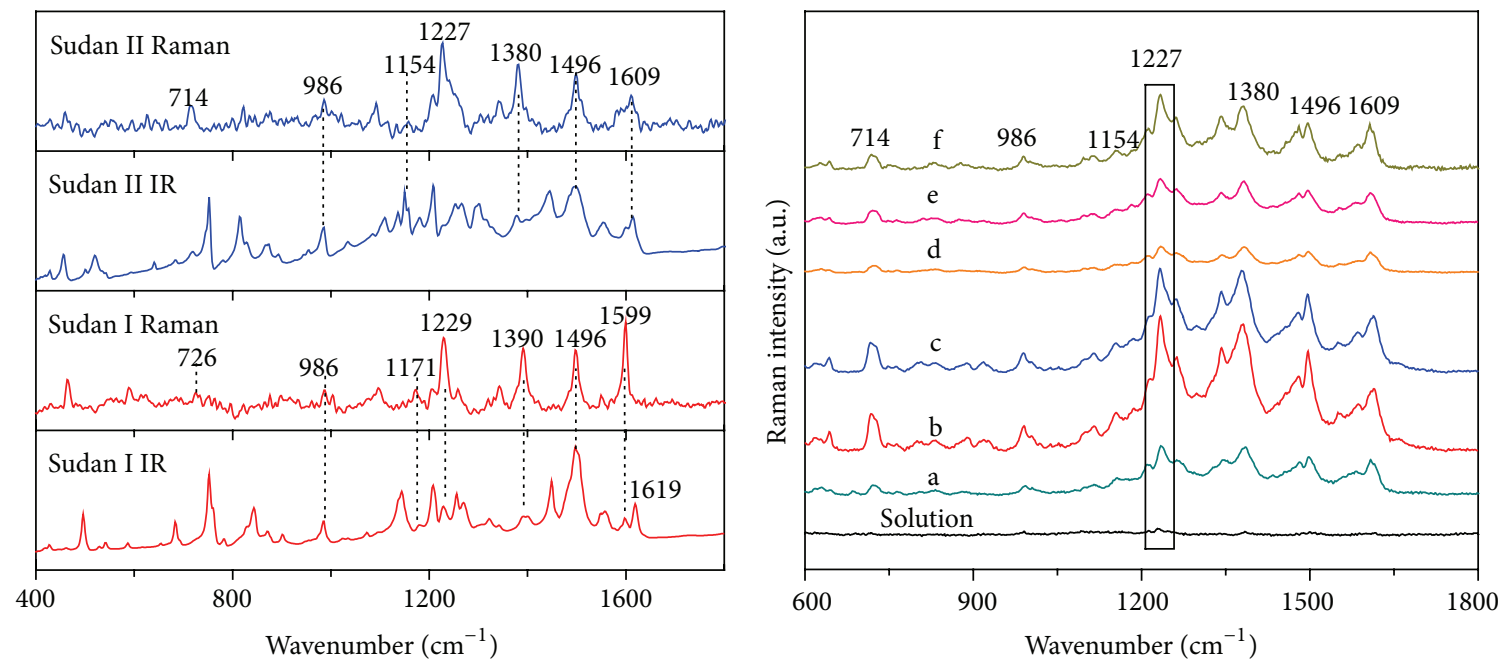

FIGURE 5: IR and Raman spectra for Sudan I and Sudan II; representative SERS spectra of 1 mg/L Sudan II solution adsorbed on Au@Ag substrates synthesized with $1 \mathrm{mmol} / \mathrm{L} \mathrm{AgNO}_{3}$ solution and various amounts of Au seeds including (a) $0.45 \mathrm{~mL}$, (b) $0.9 \mathrm{~mL}$, (c) $1.5 \mathrm{~mL}$, and (d) $4.5 \mathrm{~mL}$, as well as with $0.9 \mathrm{~mL}$ Au seeds and different concentrations of $\mathrm{AgNO}_{3}$ solutions including (e) $0.5 \mathrm{and}$ (f) $2 \mathrm{mmol} / \mathrm{L}$.

For the colloid of Au seeds, only one SPR peak was observed at $521 \mathrm{~nm}$, which was in the typical range of $517-575 \mathrm{~nm}$ for Au NPs. For the colloids of bimetallic NPs, the SPR peaks gradually red-shifted from $423 \mathrm{~nm}$ to $432 \mathrm{~nm}$ and then $446 \mathrm{~nm}$ when the amount of Au seeds used decreased from $4.5 \mathrm{~mL}$ to $0.9 \mathrm{~mL}$. The SPR peaks of the $573 \mathrm{~nm} \mathrm{Au@Ag}$ bimetallic NPs were close to that of the Ag NPs generally within the range of $390-500 \mathrm{~nm}$, indicating that the optical properties of these NPs were dominated by Ag shells [16]. As the particle sizes were further enlarged to about $120 \mathrm{~nm}$, the peak intensity declined because of the reducing concentration of ions in really large-sized Au@Ag sol, and a shoulder peak became visible at $407 \mathrm{~nm}$ due to a different electron oscillation pattern from the dipole resonance of the primary peak [17].

3.2. Raman Spectra of Sudan I and Sudan II. The similarity of Sudan I and Sudan II in their molecular structures results in similar Raman spectral features as shown in Figure 5. The spectra of two chemicals had two prominent peaks at around 1496 and $1227 \mathrm{~cm}^{-1}$ due to the stretching of double benzene ring and the $\mathrm{C}-\mathrm{H}$ in-plane bending of phenyl groups $[18,19]$.

Although Sudan I and Sudan II had similar Raman spectral features, each Sudan dye showed spectral singularities because of its unique molecular structure. The relative intensity of the peak at around $1599 \mathrm{~cm}^{-1}$ due to the stretching of $\mathrm{N}=\mathrm{N}$ bond varied between two Sudan dyes, and the absorption intensity of Sudan II at around $1609 \mathrm{~cm}^{-1}$ was relatively weak compared to Sudan I $[19,20]$. In addition, the peaks at around $1390 \mathrm{~cm}^{-1}$ in Sudan I and $1380 \mathrm{~cm}^{-1}$ in Sudan II are attributed to the $\mathrm{C}-\mathrm{N}$ and $\mathrm{C}=\mathrm{C}$ stretching in benzene rings [18]. Furthermore, the peaks arising from the $\mathrm{C}-\mathrm{H}, \mathrm{O}-$ $\mathrm{H}$ in-plane bending, and benzene rings distortions appear at $1171 \mathrm{~cm}^{-1}$ in Sudan I and $1154 \mathrm{~cm}^{-1}$ in Sudan II, respectively.
3.3. Screening SERS Substrates with High Enhancement Effect for Sudan Solutions.Au@Ag NPs with different particle sizes were used as substrates to acquire SERS spectra of Sudan II $(1 \mathrm{mg} / \mathrm{L})$, and their SERS enhancement factors $(\mathrm{EF})$ were calculated based upon the following equation [21]:

$$
\mathrm{EF}=\left(\frac{I_{\text {SERS }}}{I_{\text {norm }}}\right)\left(\frac{N_{\text {norm }}}{N_{\text {SERS }}}\right),
$$

where $I_{\text {SERS }}$ and $I_{\text {norm }}$ are the measured intensity of a specific characteristic peak for SERS and normal Raman spectra collected under the same test condition with or without the use of NPs as substrate, respectively; $N_{\text {norm }}$ and $N_{\text {SERS }}$ are the corresponding number of Sudan II molecules. Based on the strongest characteristic peak at $1227 \mathrm{~cm}^{-1}$, the EFs for six $\mathrm{Au} @ \mathrm{Ag}$ NPs were estimated to be $2.4 \times 10^{4}, 4.6 \times 10^{4}, 3.2 \times$ $10^{4}, 1.8 \times 10^{4}, 2.0 \times 10^{4}$, and $2.6 \times 10^{4}$ corresponding to the particle size of $120 \pm 11,73 \pm 6,61 \pm 5,50 \pm 4,30 \pm 2$, and $94 \pm 5 \mathrm{~nm}$, respectively. The Au@Ag NPs of about $73 \mathrm{~nm}$ led to the highest enhancement effect. This is probably because Au@Ag NPs with relatively thinner Ag shell (such as the $61 \mathrm{~nm}$ Au@Ag NPs) has less intrinsic SERS activity of Ag NPs which normally can provide stronger electromagnetic enhancement than Au NPs, while Au@Ag NPs with too thick silver shell (such as the $120 \mathrm{~nm}$ NPs) are unable to provide proper or enough junctions for Sudan II molecules adsorbed onto the surfaces of Ag shells [22].

3.4. SERS Analysis of Sudan I and Sudan II with Optimal Au@Ag Substrates. The Au@Ag NPs with particles sizes of about $73 \mathrm{~nm}$ were selected as SERS substrate for analysis of a series standard solutions of Sudan I $(0.4,0.6,0.8$, and $1 \mathrm{mg} / \mathrm{L})$ and Sudan II $(0.1,0.2,0.5$, and $1 \mathrm{mg} / \mathrm{L})$ as well as for Sudan dyes in chili extracts. As shown in Figure 6, the flat signal of blank substrates indicates that the background of substrates did not interfere with the SERS spectral features of Sudan 

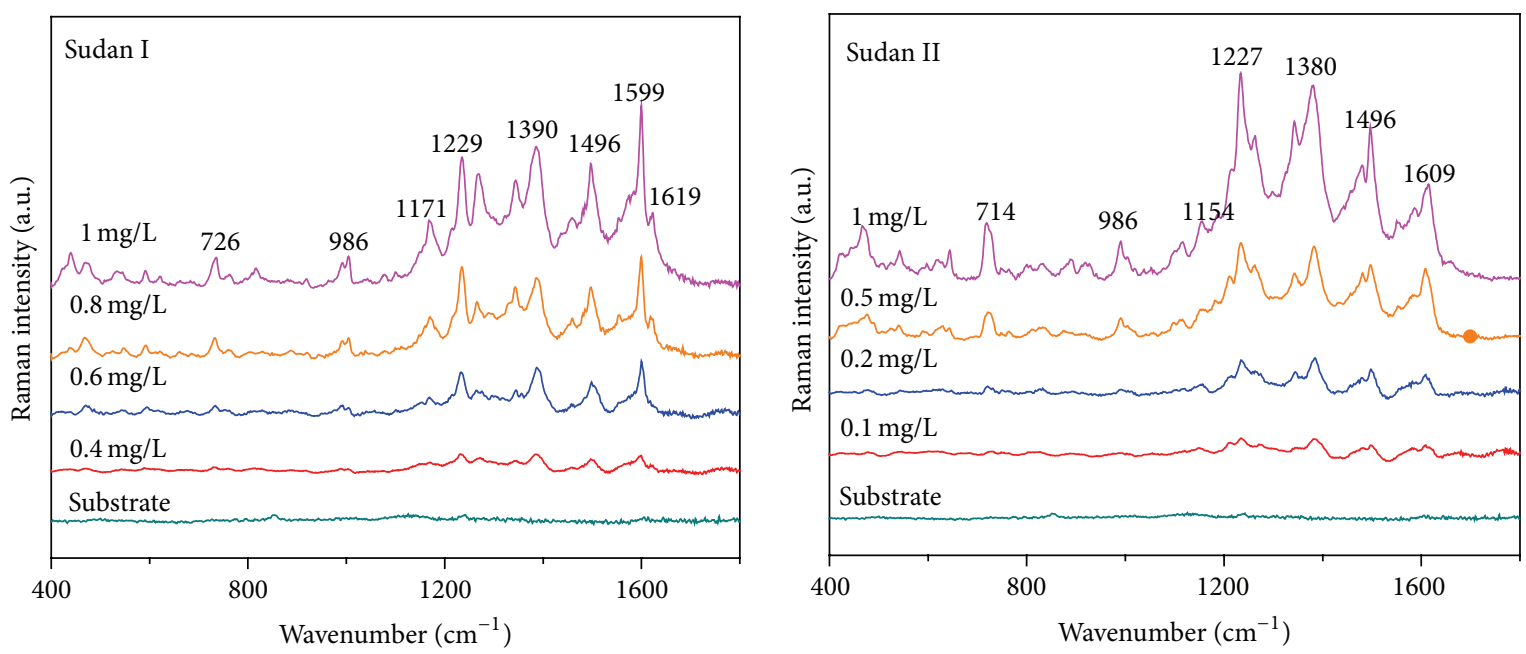

FIGURE 6: Representative SERS spectra of Sudan I and Sudan II standard solutions with Au@Ag NPs of about 73 nm as substrate.
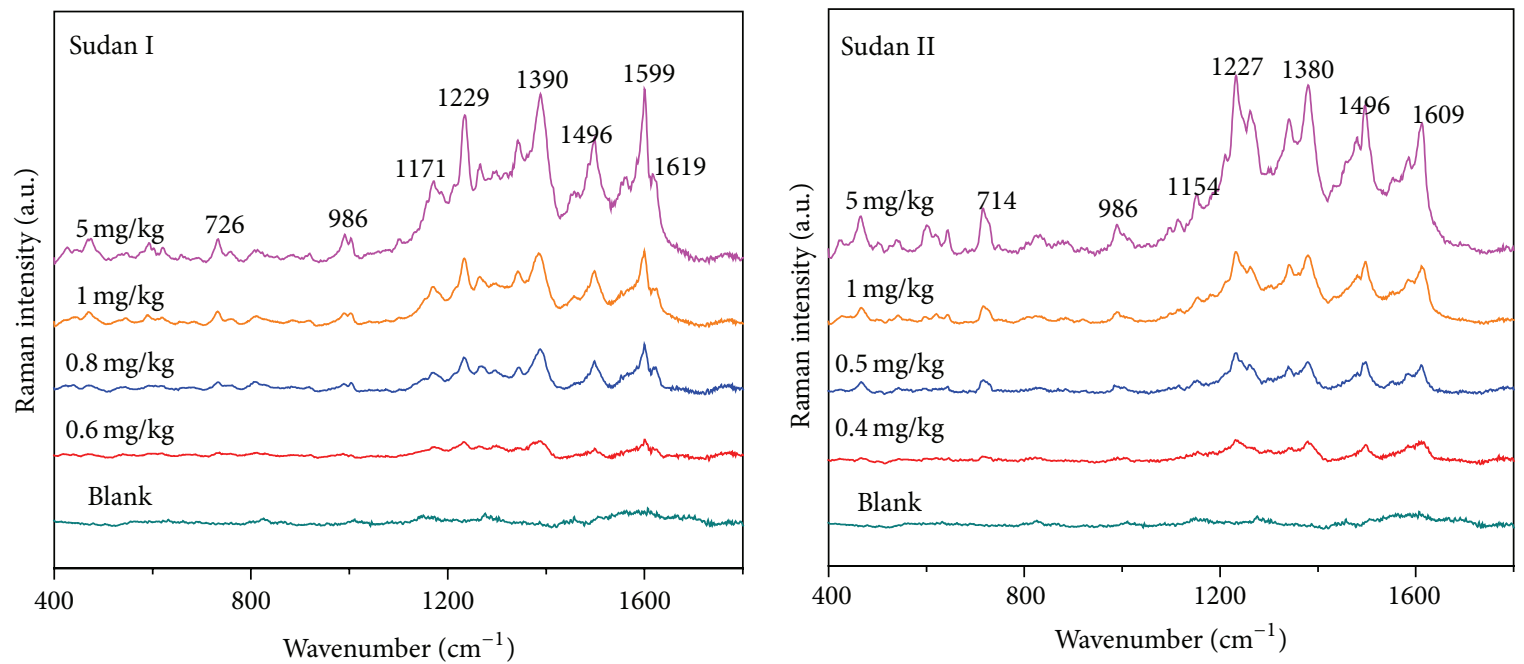

FIGURE 7: Representative SERS spectra of chili extracts spiked with Sudan I and Sudan II, using Au@Ag NPs of about 73 nm as substrate.

dyes, and the intensity of the characteristic peaks decreased as the concentration of Sudan I and Sudan II decreased. The strong and medium-strong peaks at around 1229, 1390, 1496, and $1599 \mathrm{~cm}^{-1}$ were discernible at the concentration as low as $0.4 \mathrm{mg} / \mathrm{L}$ for Sudan I and 1227, 1380, and $1486 \mathrm{~cm}^{-1}$ at $0.1 \mathrm{mg} / \mathrm{L}$ for Sudan II standards.

Figure 7 presents SERS spectra of chili extracts spiked with Sudan I [0 (blank), 0.6, 0.8, 1 , and $5 \mathrm{mg} / \mathrm{kg}$ ] and Sudan II [0 (blank), 0.4, 0.5, 1, and $5 \mathrm{mg} / \mathrm{kg}$ ], using Au@Ag NPs of about $73 \mathrm{~nm}$ as SERS substrates. No discernible SERS signals from nontargeted compounds could be found, and the major peaks in the SERS spectra of chili extracts spiked with Sudan I or Sudan II were consistent with those of their standard solutions. However, interference from chili matrices did exist since the SERS signals for chili extracts spiked with Sudan dyes were weaker than those for standard solutions with the same levels of Sudan dyes. The minimum concentration for Sudan I and Sudan II in chili extracts with major characteristic peaks discernible was $0.6 \mathrm{mg} / \mathrm{kg}$ and $0.4 \mathrm{mg} / \mathrm{kg}$, respectively, higher than their standard counterparts.

Figure 8 shows the plot of PCA $(n=36)$ scores for the first two principal components that accounted for $76.9 \%$ of the spectral variation. The chili extracts spiked with Sudan I and Sudan II could be distinguished clearly, indicating the possibility of analyzing chili powder contaminated with different types and different levels of Sudan dyes.

\section{Conclusions}

A series of sizes of Au@Ag nanospheres (diameter: 30$120 \mathrm{~nm}$ ) with good uniformity were successfully obtained without using surfactant that may hinder the effective adsorption of the analyte molecules onto the substrate surfaces. In addition, the study showed that the use of core-shell Au@Ag NPs with proper particle size (diameter: $73 \pm 6 \mathrm{~nm}$ ) and shell thickness $(27 \pm 2 \mathrm{~nm})$ as SERS substrate can 


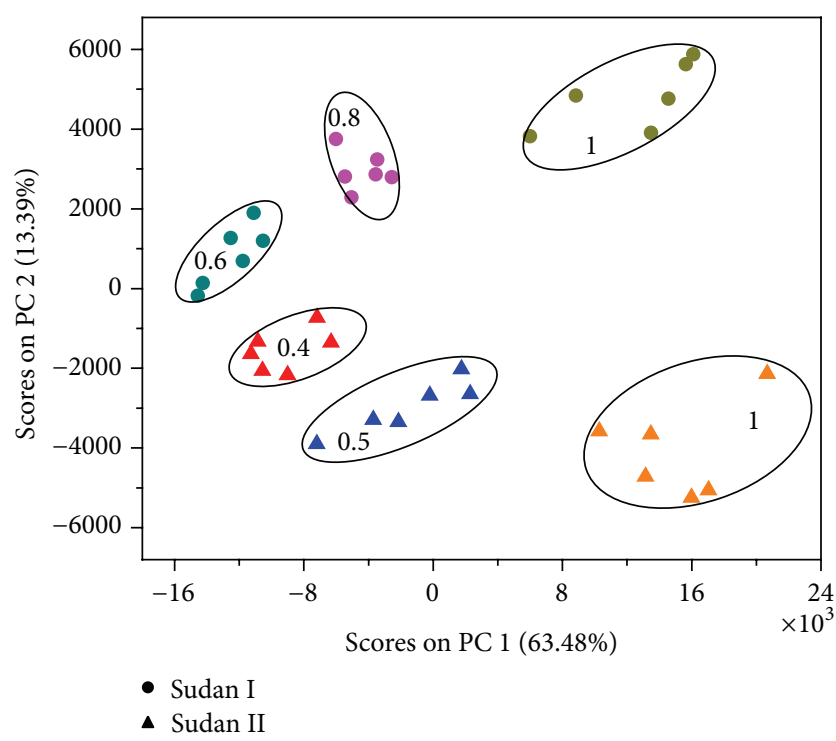

FIGURE 8: Plot of PCA score for SERS spectra of chili extracts spiked with Sudan I (0.6-1 mg/kg) and Sudan II (0.4-1 mg/kg), using Au@Ag NPs of about $73 \mathrm{~nm}$ as substrate.

greatly enhance the Raman intensities of Sudan dyes. The concentration of Sudan I and Sudan II standard solutions could be detected at levels as low as $0.4 \mathrm{mg} / \mathrm{L}$ and $0.1 \mathrm{mg} / \mathrm{L}$, respectively. In spite of some interference from the matrices extracted from chili powder, Sudan I and Sudan II in chili powder could be detected at trace levels of 0.6 and $0.4 \mathrm{mg} / \mathrm{kg}$, respectively. Finally, Sudan I and Sudan II with similar structures in complicated food matrices could be distinguished through applying PCA for analyzing SERS spectral data, indicating good selectivity of the SERS method with Au@Ag nanospheres as substrate for detection of banned additives in food stuffs at trace levels.

\section{Conflict of Interests}

The authors declare that there is no conflict of interests regarding the publication of this paper.

\section{Acknowledgments}

This research was supported by Innovation Program of Shanghai Municipal Education Commission (14YZ123) and the National Natural Science Foundation of China (61250002 and 31250006).

\section{References}

[1] International Agency for Research on Cancer, Monographs on the Evaluation of the Carcinogenetic Risk of Chemicals to Man: Some Aromatic Azo Compounds, vol. 8, International Agency for Research on Cancer, Lyon, France, 1975.

[2] H. Chen, H. Xu, O. Kweon, S. Chen, and C. E. Cerniglia, "Functional role of Trp-105 of Enterococcus faecalis azoreductase (AzoA) as resolved by structural and mutational analysis," Microbiology, vol. 154, no. 9, pp. 2659-2667, 2008.
[3] The Commission of the European Communities, "Commission decision 2003/460/EC of 20 June 2003 on emergency measures regarding hot chili and hot chili products," Official Journal of the European Communities, pp. 114-115, 2003.

[4] D. Han, M. Yu, D. Knopp, R. Niessner, M. Wu, and A. Deng, "Development of a highly sensitive and specific enzyme-linked immunosorbent assay for detection of sudan I in food samples," Journal of Agricultural and Food Chemistry, vol. 55, no. 16, pp. 6424-6430, 2007.

[5] R. A. Alvarez-Puebla and L. M. Liz-Marzán, “Traps and cages for universal SERS detection," Chemical Society Reviews, vol. 41, no. 1, pp. 43-51, 2012.

[6] A. P. Craig, A. S. Franca, and J. Irudayaraj, "Surface-enhanced Raman spectroscopy applied to food safety," Annual Review of Food Science and Technology, vol. 4, no. 1, pp. 369-380, 2013.

[7] F.-K. Liu, M.-H. Tsai, Y.-C. Hsu, and T.-C. Chu, "Analytical separation of Au/Ag core/shell nanoparticles by capillary electrophoresis," Journal of Chromatography A, vol. 1133, no. 1-2, pp. 340-346, 2006.

[8] Y. Yang, J. Liu, Z.-W. Fu, and D. Qin, “Galvanic replacement-free deposition of Au on Ag for core-shell nanocubes with enhanced chemical stability and SERS activity," Journal of the American Chemical Society, vol. 136, no. 23, pp. 8153-8156, 2014.

[9] A. K. Samal, L. Polavarapu, S. Rodal-Cedeira, L. M. LizMarzán, J. Pérez-Juste, and I. Pastoriza-Santos, "Size tunable $\mathrm{Au@Ag} \mathrm{core-shell} \mathrm{nanoparticles:} \mathrm{synthesis} \mathrm{and} \mathrm{surface-}$ enhanced Raman scattering properties," Langmuir, vol. 29, no. 48, pp. 15076-15082, 2013.

[10] J. B. Jackson, S. L. Westcott, L. R. Hirsch, J. L. West, and N. J. Halas, "Controlling the surface enhanced Raman effect via the nanoshell geometry," Applied Physics Letters, vol. 82, no. 2, pp. 257-259, 2003.

[11] L. Lu, G. Burkey, I. Halaciuga, and D. V. Goia, "Core-shell gold/silver nanoparticles: synthesis and optical properties," Journal of Colloid and Interface Science, vol. 392, no. 1, pp. 90-95, 2013.

[12] G. Frens, "Controlled nucleation for the regulation of the particle size in monodisperse gold suspensions," Nature Physical Science, vol. 241, no. 105, pp. 20-22, 1973.

[13] A. Y. Sonay, A. B. Caglayan, and M. Çulha, "Synthesis of peptide mediated Au core-Ag shell nanoparticles as surface-enhanced Raman scattering labels," Plasmonics, vol. 7, no. 1, pp. 77-86, 2012.

[14] L. Lu, H. Wang, Y. Zhou et al., "Seed-mediated growth of large, monodisperse core-shell gold-silver nanoparticles with Ag-like optical properties," Chemical Communications, no. 2, pp. 144$145,2002$.

[15] K.-T. Yong, Y. Sahoo, M. T. Swihart, and P. N. Prasad, "Synthesis and plasmonic properties of silver and gold nanoshells on polystyrene cores of different size and of gold-silver core-shell nanostructures," Colloids and Surfaces A: Physicochemical and Engineering Aspects, vol. 290, no. 1-3, pp. 89-105, 2006.

[16] H. Xu, E. J. Bjerneld, M. Käll, and L. Börjesson, "Spectroscopy of single hemoglobin molecules by surface enhanced Raman scattering," Physical Review Letters, vol. 83, no. 21, pp. 43574360, 1999.

[17] K. L. Kelly, E. Coronado, L. L. Zhao, and G. C. Schatz, "The optical properties of metal nanoparticles: the influence of size, shape, and dielectric environment," Journal of Physical Chemistry B, vol. 107, no. 3, pp. 668-677, 2003.

[18] G. R. Ferreira, H. C. Garcia, M. R. C. Couri, H. F. Dos Santos, and L. F. C. de Oliveira, "On the azo/hydrazo equilibrium in 
Sudan I azo dye derivatives," Journal of Physical Chemistry A, vol. 117, no. 3, pp. 642-649, 2013.

[19] L. Zhang, P. Zhang, and Y. Fang, "Magnetron sputtering of silver nanowires using anodic aluminum oxide template: a new active substrate of surface enhanced Raman scattering and an investigation of its enhanced mechanism," Analytica Chimica Acta, vol. 591, no. 2, pp. 214-218, 2007.

[20] R. Que, M. Shao, S. Zhuo, C. Wen, S. Wang, and S.-T. Lee, "Highly reproducible surface-enhanced raman scattering on a capillarity-assisted gold nanoparticle assembly," Advanced Functional Materials, vol. 21, no. 17, pp. 3337-3343, 2011.

[21] C. Ruan, W. Wang, and B. Gu, "Single-molecule detection of thionine on aggregated gold nanoparticles by surface enhanced Raman scattering," Journal of Raman Spectroscopy, vol. 38, no. 5, pp. 568-573, 2007.

[22] D.-K. Lim, K.-S. Jeon, H. M. Kim, J.-M. Nam, and Y. D. Suh, "Nanogap-engineerable Raman active nanodumbbells for single-molecule detection," Nature Materials, vol. 9, no. 1, pp. 60-67, 2010. 

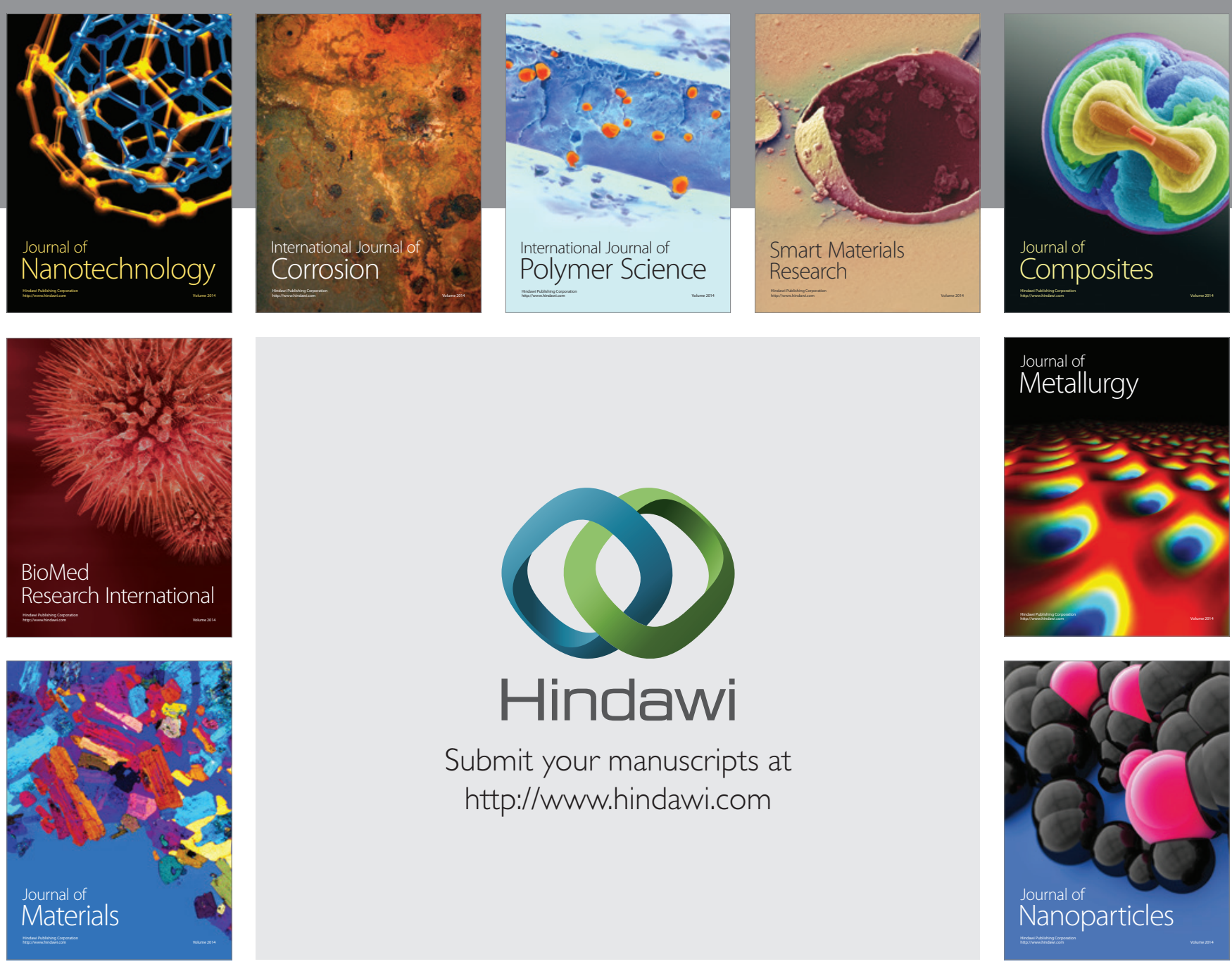

Submit your manuscripts at http://www.hindawi.com
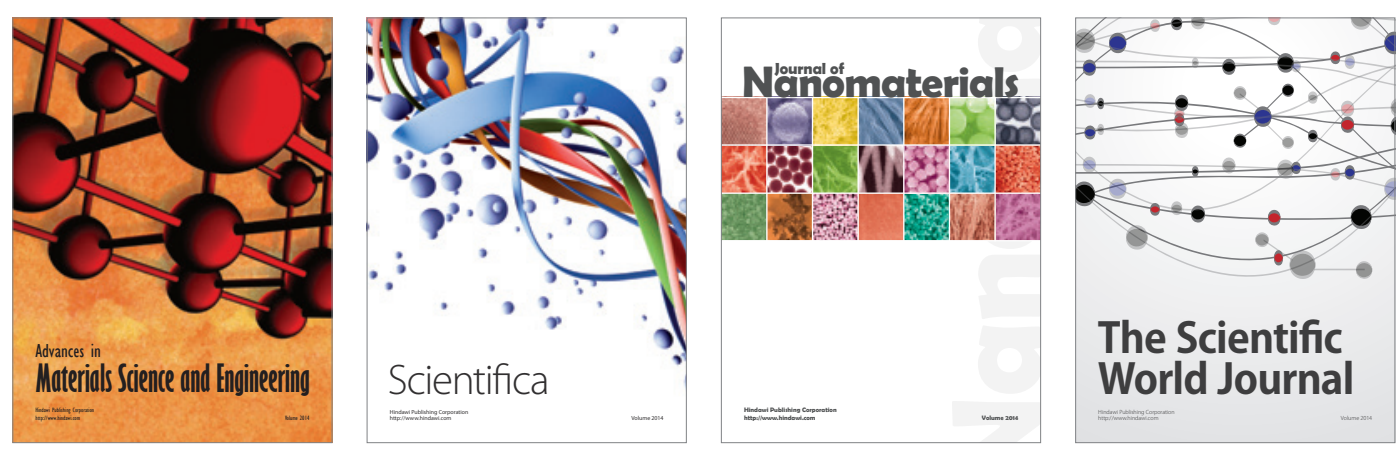

\section{The Scientific World Journal}
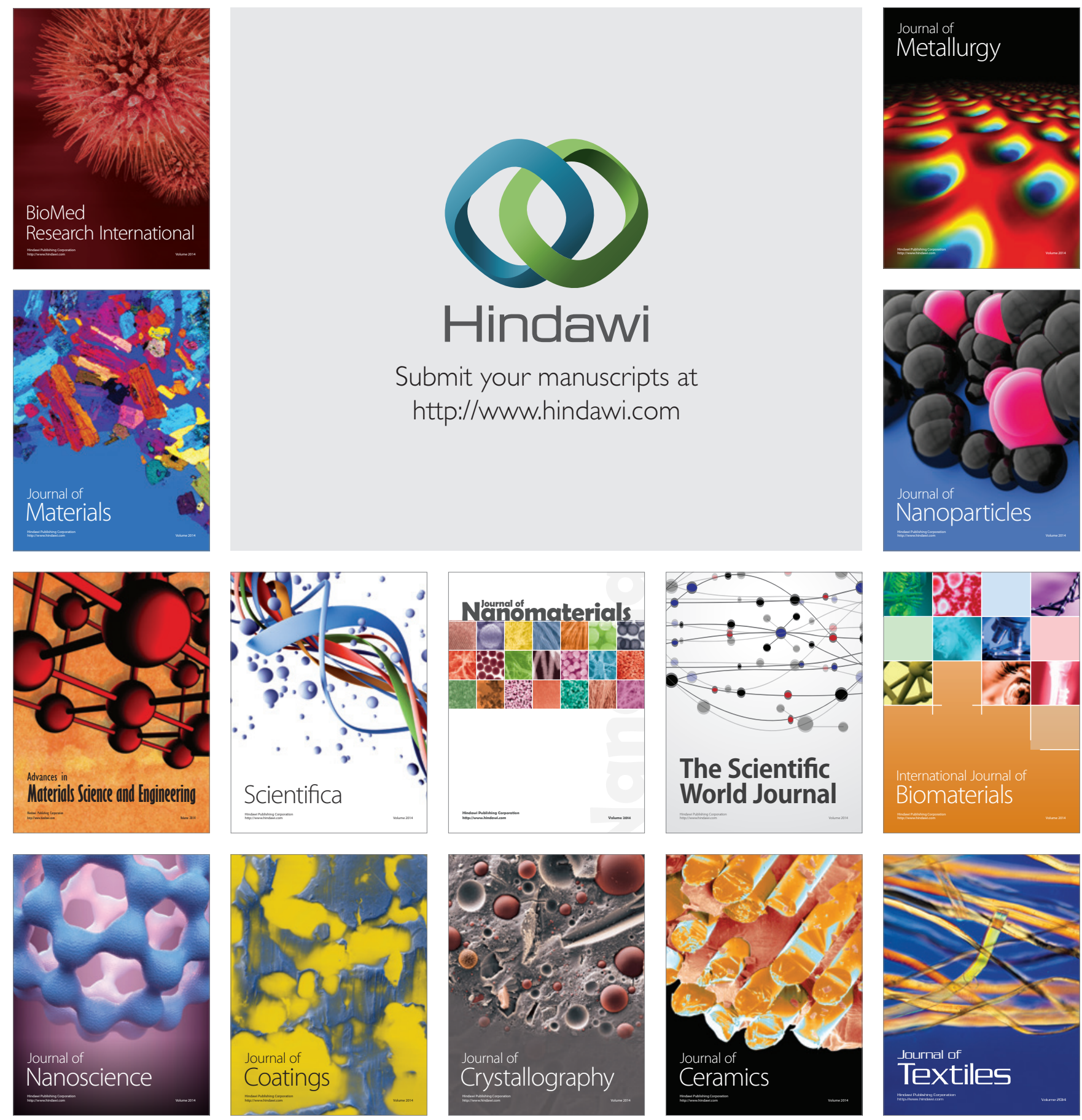